\title{
Natural Frequency and Dynamic Response of Rotor System of Steam Turbine Modelled with Euler-Bernoulli Beam
}

\author{
T. T. Deng ${ }^{1}$, J.S. Wang ${ }^{1}$, X. J. Li ${ }^{1}$, J.L. Zhong ${ }^{1}$, S.B. Wang ${ }^{1}$, \\ S. P. An ${ }^{2}$, W. G. Yang ${ }^{2}$ and D. X. Jiang ${ }^{2, a}$ \\ ${ }^{1}$ Guizhou Electric Power Test and Research Institute, Guiyang 550002, China. \\ ${ }^{2}$ State Key Laboratory of Control and Simulation of Power System and Generation Equipments, \\ Department of Thermal Engineering, Tsinghua University, Beijing 100084, China. \\ ajiangdx@tsinghua.edu.cn
}

Keywords: Natural frequency, transient response, rotor system, steam turbine, Euler-Bernoulli Beam.

\begin{abstract}
Based on Finite element method (FEM), analysis of natural frequency and dynamics response is carried out in this work. Firstly, modeling of rotor system in steam turbines is analyzed with Euler-Bernoulli beam element. Secondly, natural frequency of the rotor system is obtained and compared. Thirdly, dynamic response of the rotor system is simulated and the vibration of the rotor system is obtained. The results show that the proposed model can obtain results with high accuracy, and the model is efficient. The modeling approach presented in this work provides a fast and accurate method in analyzing dynamic characteristics of the practical rotor system in steam turbine units
\end{abstract}

\section{Introduction}

Dynamic characteristics of rotor system on natural frequency and dynamic response are important issues in design and operation of rotating machinery such as steam turbine. The rotor system of modern steam turbines with large capacity usually rotates in 3000 revolution per minute (RPM), which is higher than the first-order natural frequency [1]. It means that the rotor system needs to pass through the critical speed during the start-up and shutdown. And procedures in operation need to consider the situation to avoid the rotor system operating in the speed close to the critical speed. The critical speed is dependent on natural frequency of the rotor system and other conditions of the circumstance. Therefore, determination of natural frequency of the rotor system is important.

When the rotor system of the steam turbine runs in the rated condition, the dynamics response of the rotor system is highly related to the vibration, where the vibration is an important monitoring parameter in operation, and the level of the vibration is the key factor in evaluating the health status of the steam turbine. For example, when the amplitude of the vibration of the rotors exceeds $150 \mathrm{um}$, the steam turbine must shut down, according to the operation standard (for the vibration of the bearing, the limit is $80 \mathrm{um}$ ) [2]. Obtaining dynamic response of rotor system is beneficial in estimating the vibration level of the steam turbine.

Finite element method (FEM) is widely applied in kinds of devices to obtain solution in different purposes, including static solution for estimating stress and strain, modal analysis for calculating the natural frequency and modal shape, harmonic analysis for computing the responses with specific excitation, and transient analysis for obtaining dynamic responses in time-varying loads [3-6]. Due to its generality, FEM can be applied in obtaining natural frequency and dynamic response of rotor system of steam turbine. Furthermore, the model formed with FEM can be standardized for rotor systems in different steam turbines.

Based on FEM, analysis of natural frequency and dynamics response is carried out in this work. Firstly, modeling of rotor system in steam turbines is studied, where the element is formed with Euler-Bernoulli beam. Secondly, natural frequency of the rotor system is obtained and compared. Thirdly, dynamic response of the rotor system is simulated and the vibration of the rotor system is obtained. 


\section{Methods and Models}

Euler-Bernoulli Beam Element. The Euler-Bernoulli beam model is shown in Fig. 1. The assumption used in Euler-Bernoulli beam is that the section is perpendicular to the center of the beam after the deformation. That is to say, shear deformation is excluded in Euler-Bernoulli beam model. With the assumption, the motion of equation is:

$$
\rho \mathrm{A} \frac{\partial^{2} v}{\partial \mathrm{t}^{2}}+\frac{\partial^{2}}{\partial x^{2}}\left(E I \frac{\partial^{2} v}{\partial x^{2}}\right)=q(x, t),
$$

where $v(x, t)$ is the displacement along the $\mathrm{x}$ direction, $\rho$ is the mass density of the beam, $A$ is the area of the section, $E$ is the elastic modulus of the material, $I$ is the inertia of the beam, $q(x, t)$ is the load acted on the beam, $t, x$ are the time and coordinate along the center of the beam, respectively.

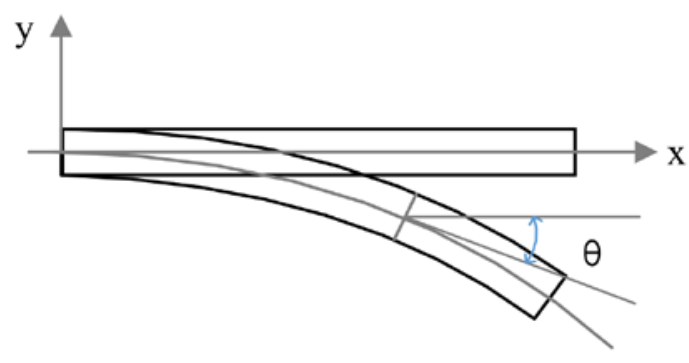

Figure 1. Euler-Bernoulli beam

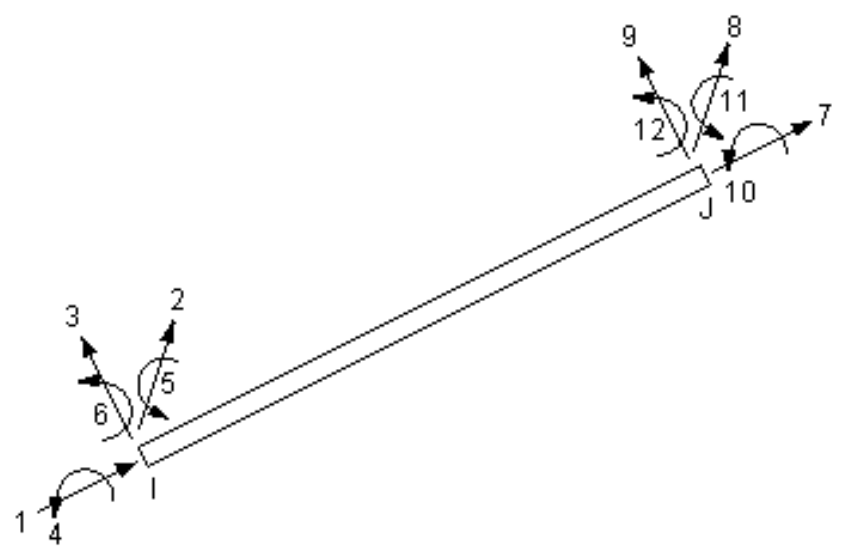

Figure 2. DOFs defined in Euler-Bernoulli beam element

With Euler-Bernoulli beam model, Euler-Bernoulli beam element is formed with Finite Element Method (FEM). Two nodes are applied in defining the Euler-Bernoulli beam element, where 6 Degrees of Freedom (DOFs) are included in each node. The 6 DOFs include three DOFs in displacements (UX, UY, UZ), and three DOFs in rotation (ROTX, ROTY, ROTZ). The DOFs defined in Euler-Bernoulli beam element are shown in Fig. 2.

With the defined DOFs, the stiffness matrix and the mass matrix of the Euler-Bernoulli beam element can be derived.

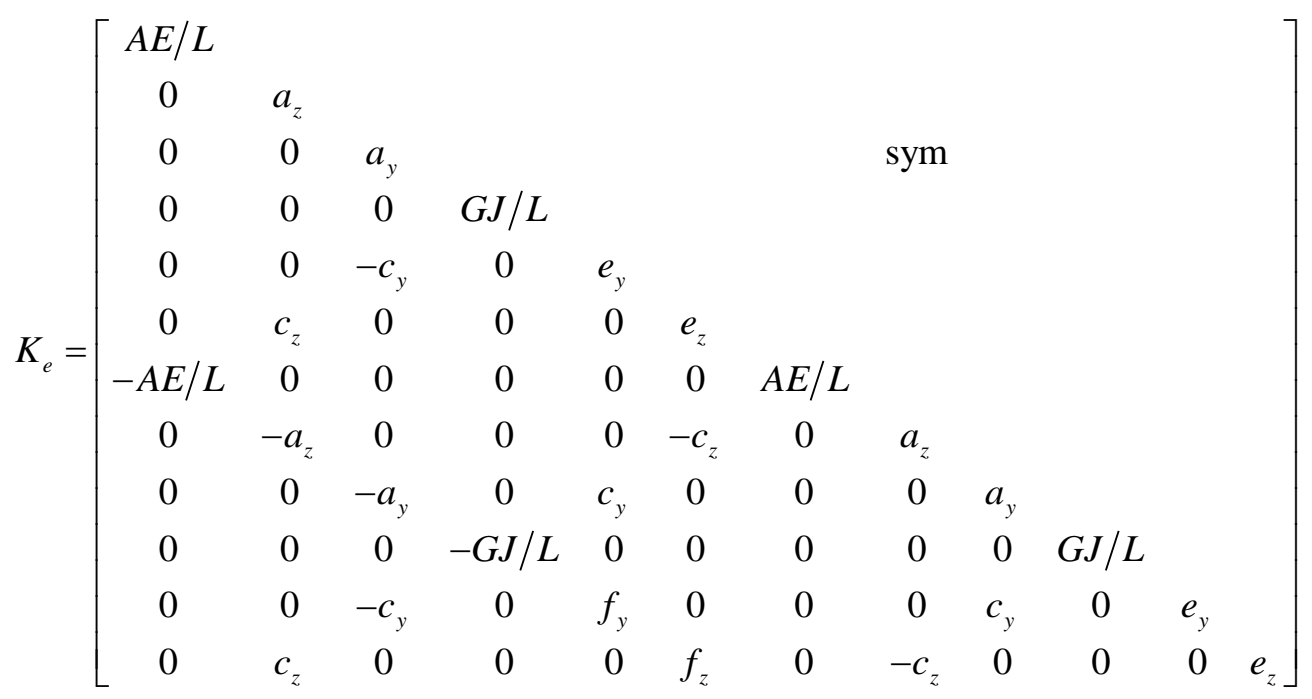

where, $A$ is the area of the section, $\mathrm{L}$ is the length of the element, $\mathrm{G}$ is the shear modulus, $I_{X}$ is the inertia, $\mathrm{J}$ is the torque. 


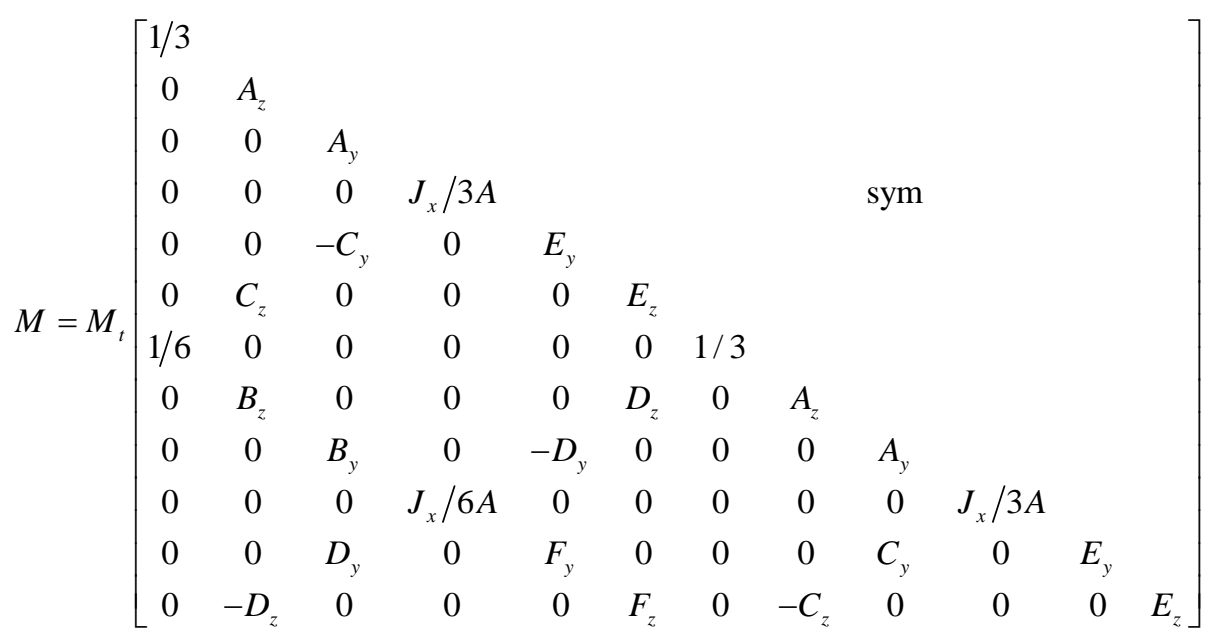

where $M_{t}=(\rho A+m) L\left(1-\varepsilon_{\text {in }}\right)$, $\mathrm{m}$ is the additional mass per length, and $\varepsilon_{\text {in }}$ is the prestress.

Damping matrix caused by gyroscopic effect is defined:

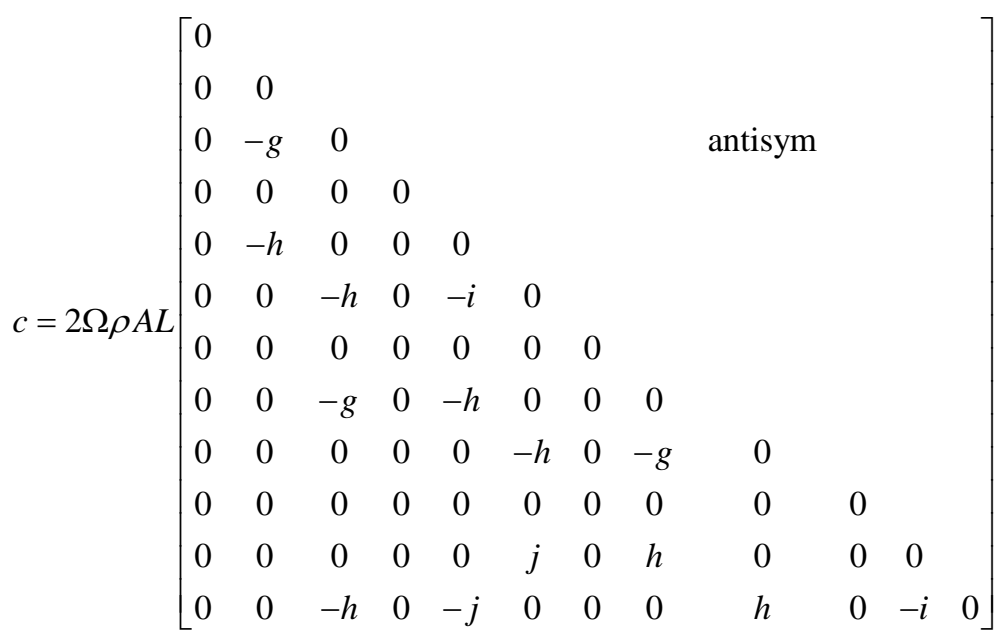

where, $\Omega$ is the rotational speed along $\mathrm{X}$ axis,

$$
\begin{aligned}
& g=\frac{6 / 5 r^{2}}{L^{2}(1+\phi)^{2}}, \\
& h=\frac{-(1 / 10-1 / 2 \phi) r^{2}}{L(1+\phi)^{2}}, \\
& i=\frac{\left(2 / 15+1 / 6 \phi+1 / 3 \phi^{2}\right) r^{2}}{(1+\phi)^{2}}, A^{S}=A^{W} / 2.0 \\
& j=\frac{-\left(1 / 30+1 / 6 \phi-1 / 6 \phi^{2}\right) r^{2}}{(1+\phi)^{2}}, r=\sqrt{I / A}, \phi=\frac{12 E I}{G A^{S} L^{2}}, A=A^{W}=\frac{\pi}{4}\left(D_{0}^{2}-D_{i}^{2}\right), \\
& I_{y}=I_{z}=I=\frac{\pi}{64}\left(D_{0}^{4}-D_{i}^{4}\right), J=\frac{\pi}{32}\left(D_{0}^{4}-D_{i}^{4}\right) .
\end{aligned}
$$

Model of Rotor System with Euler-Bernoulli Beam. The scheme of the rotor system in a 660MW steam turbine generator is shown in Fig. 3.

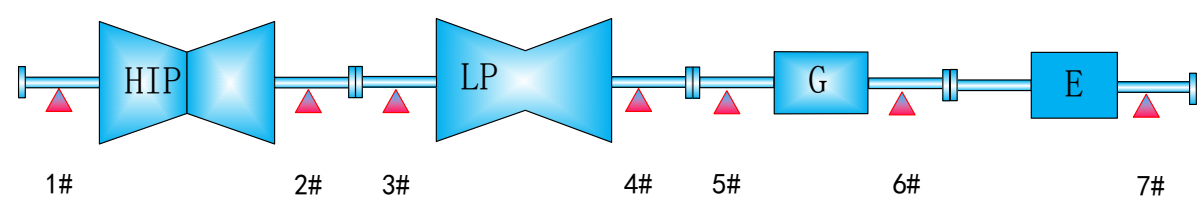

Figure. 3 The scheme of the rotor system in a 660MW steam turbine generator 
With Euler-Bernoulli beam element, the rotor system is modelled. In this work, 162 elements are applied in the model of the rotor system. Also, the bearings are modelled as springs with stiffness and damping. The parameters used in bearings are based on the practical situation.

Finally, the model of the rotor system is obtained, and the matrices of the stiffness, the mass and the damping are assembled with the elements' matrices.

With the loads exerted on the rotor system, the motion of equation of the rotor system can be expressed as,

$$
M \ddot{U}+C \dot{U}+K U=F(t) .
$$

Newmark Integration Scheme for Transient Solution. To solve the motion of equations obtained in the previous section, Newmark integration scheme is applied.

$$
\left[d_{0} \mathbf{M}+d_{1} \mathbf{C}+\mathbf{K}_{n}\right] \mathbf{U}_{n+1}=\mathbf{F}\left(t_{n+1}\right)+\mathbf{M}\left(d_{0} \mathbf{U}_{n}+d_{2} \dot{\mathbf{U}}_{n d}+d_{3} \ddot{\mathbf{U}}_{n}\right)+\mathbf{C}\left(d_{1} \mathbf{U}_{n}+d_{4} \dot{\mathbf{U}}_{n}+d_{5} \ddot{\mathbf{U}}_{n}\right),
$$

where, $\quad d_{0}=\frac{1}{\alpha(d t)^{2}}, d_{1}=\frac{\delta}{\alpha d t}, d_{2}=\frac{1}{\alpha d t}, d_{3}=\frac{1}{2 \alpha}-1, d_{4}=\frac{\delta}{\alpha}-1, d_{5}=\frac{d t}{2}\left(\frac{\hat{\delta}}{\alpha}-2\right)$

The parameters of time integration consist of $\mathrm{dt}, \alpha$, and $\delta$. The relationship of $\alpha$ and $\delta$ follows:

$$
\delta=\frac{1}{2}+\gamma, \alpha=\frac{1}{4}(1+\gamma)^{2}, \gamma>0
$$

\section{Results and Discussions}

Natural Frequency of Rotor System. The natural frequencies of the rotor system can be obtained with the established model. The natural frequencies of the rotor system in the lateral direction are listed in Table 1, where the results obtained with Ansys are also listed for comparison.

Table 1. The natural frequencies of the rotor system in the lateral direction

\begin{tabular}{c|c|c|c}
\hline Order & Ansys & $\begin{array}{c}\text { Model in this } \\
\text { work }\end{array}$ & Error (\%) \\
\hline 1 & 13.175 & 13.322 & 1.12 \\
2 & 19.593 & 20.16 & 2.88 \\
3 & 27.158 & 27.68 & 1.91 \\
4 & 33.284 & 34.10 & 2.47 \\
5 & 52.834 & 54.53 & 3.21 \\
6 & 54.206 & 57.40 & 5.90 \\
7 & 59.422 & 61.20 & 2.99 \\
8 & 89.885 & 94.58 & 5.23 \\
9 & 104.39 & 106.76 & 2.27 \\
10 & 120.58 & 127.43 & 5.68 \\
11 & 123.71 & 131.12 & 5.99 \\
\hline
\end{tabular}


From the results listed in Table 1, the natural frequency obtained with the model in this work is consistent with that obtained with Ansys, where the error is less than 6\%. It shows that the model formed in this work obtains high accuracy and is suitable for analysis of natural frequency of the rotor system in steam turbine unit.

Dynamic Responses of Rotor System. Dynamic responses are obtained with Newmark integration scheme shown in the previous section. The results of the rotor system in the normal condition are shown in Figs. 4-5, where the rotating speed is 3000RPM.

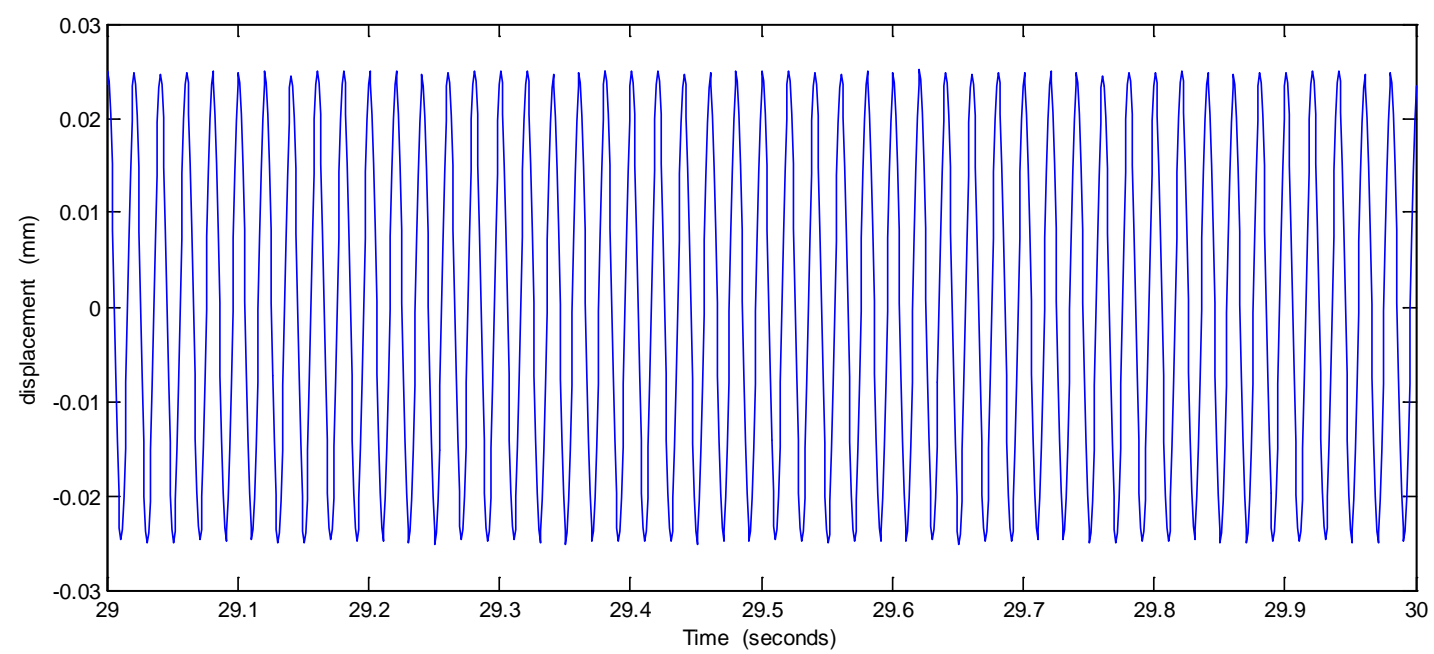

Figure. 4 Dynamic responses of the rotor system in normal condition (displacements)

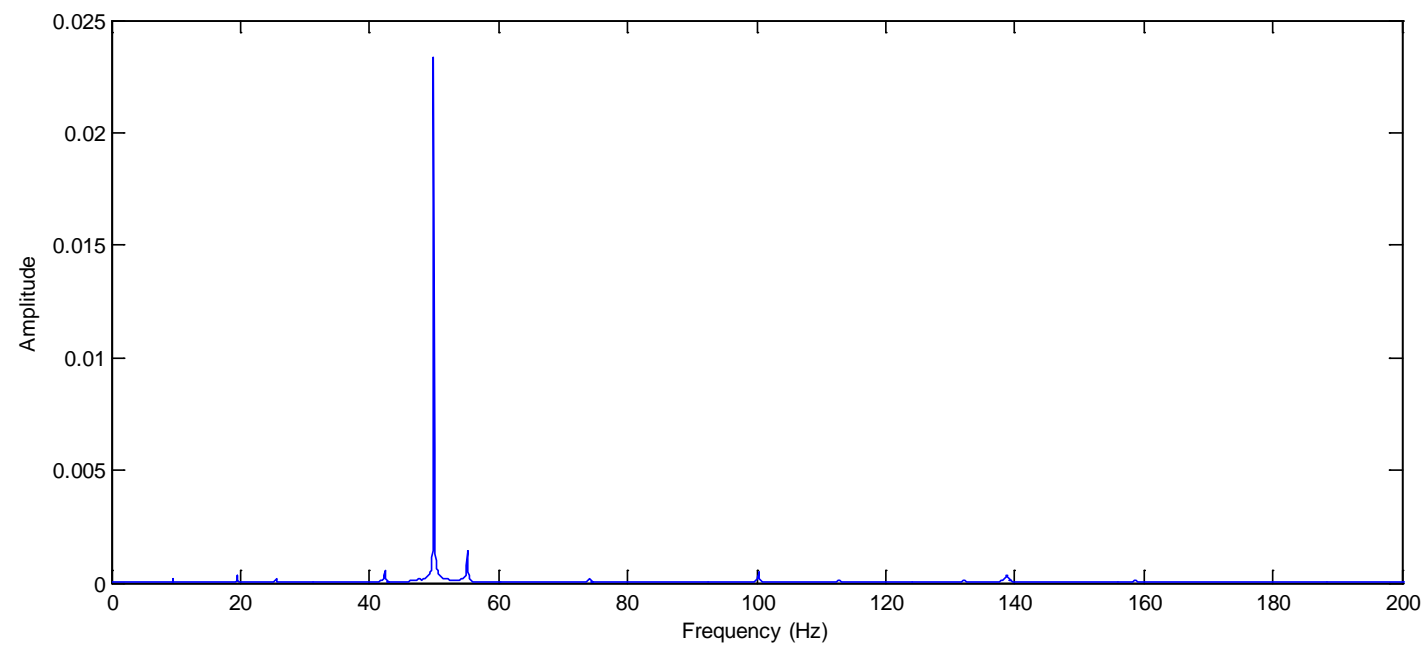

Figure. 5 Spectrum analysis of vibration in the rotor system in normal condition

Discussions. Natural frequency and dynamic response are calculated in this work, using the model formed by Euler-Bernoulli beam elements. The results show that the proposed model can obtain results in high accuracy. And the model is efficient.

For the application in reality, the modeling approach with Euler-Bernoulli beam element can simulate the dynamic responses of the start-up and shutsown to study the vibraiton level when the rotor system passes through the critical speed. It can also be used in simulating the dynamic respones of the rotor system in typical faults, such as unbalance, misalignment, rub, and torsional excitation.

The dynamic responses of the rotor systemin fault condition can be used to study the vibational features of the faults, which is beneficial for formulation of diagnostic rules. As the vibrational features are based on the practical rotor system with specific parameters, the obtained rules will be more accurate than that obtained by Jeffcott rotor model. 
Further study is being carried out in modeling different fault conditions and obtaining vibrational features in fault conditions.

\section{Conclusion}

With Euler-Bernoulli beam element, rotor system in 600MW steam turbine generator is modelled. Then natural frequencies and dyanmic responses of the rotor system are analyzed. The results show that the proposed model can obtain results in high accuracy. And the model is efficient. The modeling approach presented in this work provides a fast and accurate method in analyzing dynamic characteristics of the practical rotor system in steam turbine units, which is beneficial for formulation of diagnostic rules and improve the accuracy of the fault diagnosis. Further study is being carried out in modeling different fault conditions and obtaining vibrational features in fault conditions.

\section{References}

[1] Jiang, Dongxiang, Liangyou Hong, Zheng Wang, and Xiaorong Xie. "Torsional Vibration Analysis and Stress Calculation for the Fault 600MW Steam Turbine Generator Shaft System." In ASME 2009 International Design Engineering Technical Conferences and Computers and Information in Engineering Conference, pp. 103-109. American Society of Mechanical Engineers, 2009.

[2] Meng, Li, Shu Yunxing, Mao Jiandong, and Li Xiaohua. "Extraction Fault Rule of Rotation Equipment Based on Rough Set." In Intelligent Computation Technology and Automation, 2009. ICICTA'09. Second International Conference on, vol. 2, pp. 604-607. IEEE, 2009.

[3] Dhatt, Gouri, Emmanuel Lefrançois, and Gilbert Touzot. Finite element method. John Wiley \& Sons, 2012.

[4] Zienkiewicz, Olgierd Cecil, and Robert Leroy Taylor. The finite element method: Solid mechanics. Vol. 2. Butterworth-heinemann, 2000.

[5] Torkaman, Hossein, Ebrahim Afjei, and Peyman Yadegari. "Static, dynamic, and mixed eccentricity faults diagnosis in switched reluctance motors using transient finite element method and experiments." Magnetics, IEEE Transactions on 48, no. 8 (2012): 2254-2264.

[6] Rao, Singiresu S. The Finite Element Method in Engineering: Pergamon International Library of Science, Technology, Engineering and Social Studies. Elsevier, 2013. 